\title{
Primary myxoma of the aorta
}

\author{
J. GOUGH' ${ }^{1}$ AND W. MOREANO
}

From the Department of Pathology, Stanford University Medical Center, Stanford, California

SYNOPSIS A case of primary myxoma of the aorta is described in a 62-year-old woman. The patient died following surgery in which a teflon graft was inserted to bypass the tumour which had caused partial obstruction of the ascending aorta. The tumour was limited to the subintima and had not metastasized.

Primary tumours of the aorta are very rare. Sarcomata may arise in the wall of the aorta (Nencki, 1946), while some tumours are intraluminal (Karhoff, 1952; Kovalena and Press, 1959; Kattus, Longmire, Cannon, Webb, and Johnston, 1960). The case described here is that of an intraluminal myxoma causing obstruction to the arteries arising from the aortic arch, in which surgery was complicated by multiple emboli.

\section{Clinical Summary}

A 62-year-old Caucasian woman was admitted to Stanford University Hospital in December, 1970, with a year's history of headaches, dizziness, and blackouts which had become worse during the previous month. Her referring practitioner had stated that she had been hypertensive for three to four years but that the blood pressure had recently been normal. Past history included a hysterectomy for fibroids in 1952.

Physical examination showed a confused patient with finger clubbing, weak carotid pulses, no pulses in the right arm, a weak left brachial pulse, and normal femoral pulses. Blood pressure was $100 / 80$ in the left arm and $160 / 80$ in the legs. Tendon reflexes were increased in the legs and the plantar responses were extensor.

An arch aortogram showed unusual features simulating a dissecting aneurysm of the ascending aorta. (The radiological details have been reported elsewhere by Silverman and Wexler, 1972.) The innominate and left carotid arteries were totally occluded; the right carotid, both vertebral and left subclavian arteries showed localized narrowing.

An intraaortic tumour was found at thoracotomy

${ }^{1}$ Present address: Department of Pathology, Welsh National School of Medicine, Heath Park, Cardiff

Received for publication 12 July 1974. and a teflon graft was placed between the aortic arch and the innominate and left carotid arteries to bypass the occlusion. During the operation tumour embolized to the femoral arteries and the emboli were removed. The patient failed to regain consciousness, experienced increasingly frequent convulsions and died three days after the operation.

\section{Necropsy Findings}

A thrombus-like plaque of tumour, $12 \times 4 \mathrm{~cm}$, was present in the ascending aorta and aortic arch? (fig 1), extending into the left carotid artety with almost complete occlusion, and causing partial occlusion of the innominate $(70 \%)$ and left subclavian $(20 \%)$ arteries. No gross evidence of invasion into the aortic wall was present. Severe atheroma was present in the thoracic aorta underlying the tumour. The teflon bypass graft from the ascending aorta to

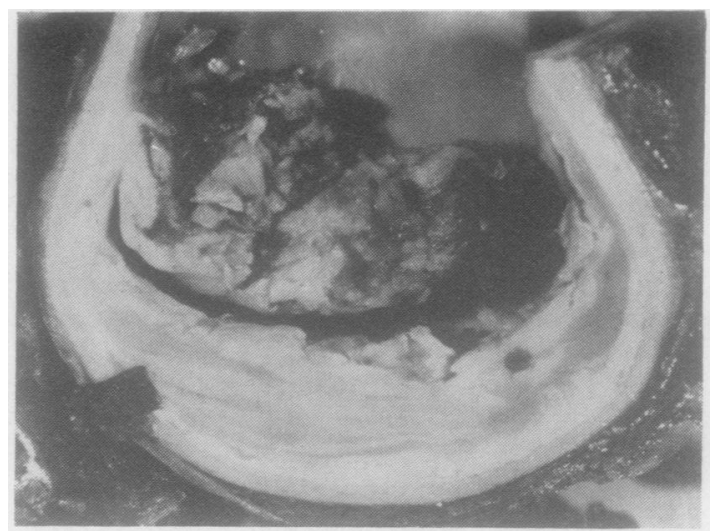

Fig 1 Transverse section of aorta showing intraluminal tumour and thrombus. $\times 2$. 
the innominate and left carotid arteries was patent and its anastomoses were intact.

The heart weighed $500 \mathrm{~g}$ and the left ventricle was hypertrophied. The anterior descending branch of the left coronary artery showed severe atherosclerosis, while mild atheroma was present in the circumflex and right coronary arteries. A recent infarct was present in the liver and in the spleen ( $2 \mathrm{~cm}$ diameter), and both kidneys contained several infarcts up to $4 \mathrm{~cm}$ in diameter. A large recent infarct was present in the left parietal lobe, and a smaller older infarct was seen in the left striate cortex. Subtotal hysterectomy was noted; other organs were grossly normal.

Microscopy of the aorta and neck vessels showed a tumour which appeared to have arisen in the intima or subintima and grown into the lumen of the various blood vessels, but there was no evidence of invasion of the media at any point. The tumour was partially covered by thrombus and mostly necrotic, but where viable was composed of oval, round and spindle shaped cells with nuclei $10-20 \mu$ in diameter (fig 2 ).

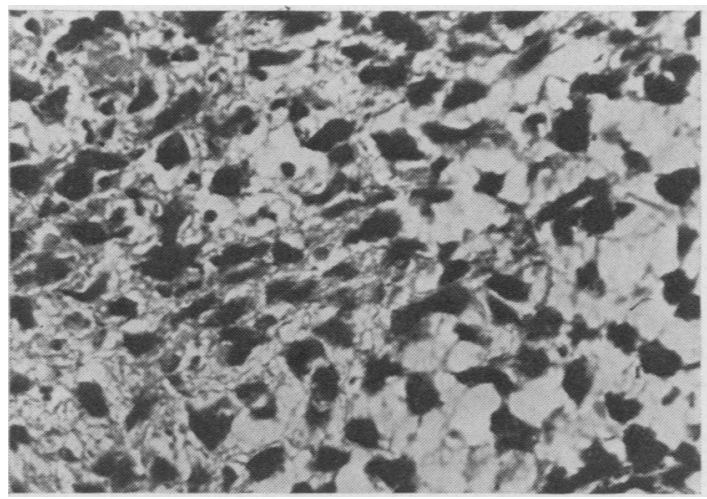

Fig 2 Section of aortic tumour showing pleomorphic stellate cells. Haematoxylin and eosin. $\times 250$.

An occasional nucleolus was seen; mitoses were infrequent. Some cells had little cytoplasm, others had more and where the texture of the tumour was loose many cells were seen to have dendritic processes. A reticulin stain showed an extensive reticulin fibre network, surrounding almost every tumour cell (fig 3). Van Gieson staining showed a little collagen at the base of the tumour, and Alcian blue and colloidal iron stains showed considerable amounts of extracellular mucopolysaccharide, which also stained weakly with periodic-acid-Schiff, and the appearances were considered to represent a myxoma. No metaplastic or heterologous elements were identified in many sections examined.

Other microscopic findings included the con-

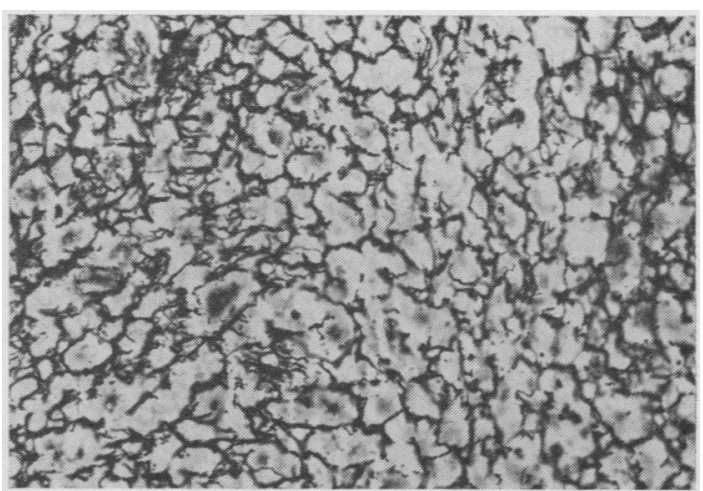

Fig 3 Section of aortic tumour showing abundant reticulin fibre formation. Reticulin stain. $\times 250$.

firmation of recent infarction in the brain, liver, spleen, and kidney and areas of fresh necrosis in the adrenals. Only one example of arterial occlusion was demonstrated in the various infarcts, and no evidence of tumour was demonstrated in the thrombus occluding this artery.

\section{Discussion}

Very few examples of primary intraluminal aortic tumours have been reported. The tumour recorded by Karhoff (1952) was considered to be of endothelial origin, causing death by occlusion of the superior mesenteric artery. Kovalena and Press (1959) described as a case of sarcoma of the aortic intima which metastasized to the pelvis. The fibromyxoma reported by Kattus et al is similar to the present case with the exception that the former contained metaplastic cartilage and bone. The patient presented with aortic obstruction and the tumour was successfully removed.

The myxoma reported here is likely to be a benign tumour, or at most of low-grade malignancy. No invasion of the media was present nor was there evidence of metastases. The tumour therefore shows a greater resemblance to a cardiac myxoma than to a soft tissue myxoma which commonly results in local infiltration.

\section{References}

Karhoff, B. (1952). Primärtumor der Aorta. (Abstr.) Zbl. allg. Path. path. Anat., 89, 46.

Kattus, A. A., Jr., Longmire, W. P., Cannon, J. A., Webb, R., and Johnston, C. (1960). Primary intraluminal tumor of the aorta producing malignant hypertension. New Engl. J. Med., 262, 694-700.

Kovalena, A. N., and Press, B. O. (1959). A case of primary sarcoma of the intima of the aorta. Arkh. Pat., 21 (10), 62-65.

Nencki, L. (1946). Zur Kenntnis der Primärtumoren der grossen Gefässstämme: ueber einen Fall von primären Sarcoma der Aorta abdominalis. Cardiologia, 10, 1-24.

Silverman, J. F., and Wexler, L. (1972). Primary intraluminal tumor of the aorta. Radiology, 102, 581-582. 